\title{
Performance Analysis of Satellite-Terrestrial AF Cooperative System at Venezuelan Amazon Region
}

\author{
G. Guedez, Q. Gao, and J. Uscategui
}

\begin{abstract}
A significant quantity of the Venezuelan satellite communications services operate at Ka band for the Amazonian Region. The Ka Band signals are exposed to severe quality degradation and unacceptable error rate capabilities due to climate constrains. In order to improve the error rate performance, an Amplify and Forward (AF) cooperative scheme is proposed. The basic principle is based on the relay of multiple scaled version of the received signal at the destination, which can be optimally combined at the receiver using a Maximum Ratio Combiner (MRC). The performance of the satellite-terrestrial AF cooperative system is compared with the direct satellite transmission system by simulation.
\end{abstract}

Index Terms-AF, BER, Ka Band, MRC, SNR.

\section{INTRODUCTION}

The Venezuelan Amazonian hold spectacular natural wonders and its rivers represent a significant percentage of the world fresh water reserve. Thus, the construction of wire guide communication system is not feasible, according to international environment regulation. In order to protect the Amazonian ecosystem, the satellite communication systems represent the best method to provide communications services at the Amazonian area. However, the signal in $\mathrm{Ka}$ band are sensible to degradation due to the environment conditions. In some cases, the link quality is not sufficient to deliver data successfully [1] and [2]. The AF cooperative communication principle can be useful to combat the problem. The improvement relies on the transmission of multiple copies from the same signal and combine optimally the receive signals using MRC. Thus, even if one copy of the signal has not sufficient quality, the probability that all the copies has poor quality is very low [3]. The relays nodes should amplify and forward copies of the signal independently to each other. The probability that all the signals face simultaneously bad propagation conditions is low. The AF cooperative gain is analyzed over the transmission channel. The satellite communication channel follow a Rice distribution [3] and [4]. The channel between the relay nodes and the destination (terrestrial communication) is also considered as a Rice distribution. The Rice distribution consist of a strong component of line of sight (LOS), combined with a weaker multipath or non-line of sight (NLOS) [4]. The non-line of sight (NLOS) component can be neglected because the ground station antenna has a large gain. The main purpose of the research, is to analyze the system bit error rate for $\mathrm{Ka}$ Band satellite signals in cooperative mode, and compare the results with the

Manuscript received January 16, 2017; revised May 1, 2017.

The authors are with the School of Electronic and Information Engineering, Beihang University, China (e-mail: gaoqiang@buaa.edu.cn). performance of classic satellite communication mode.

\section{SYSTEM MODEL}

\section{A. AF Cooperative Scheme}

Consider the scheme shown in the Fig.1. The Venezuelan satellite transmit to several earth stations. All the stations have high gain fixed antennas, this means the NLOS components is close to zero.



Fig. 1. VENESAT-1 AF cooperative scheme.

The Venezuelan satellite represent the source. The channel between the satellite and each earth station follow a Rician Distribution. The earth stations can communicate each other using high directive antennas. The channel between earth stations is considered a Rician channel with $K=1$.

In Phase I, the source (VENESAT) transmit with a Power $\mathrm{P}_{\mathrm{S}}$, a symbol block $\mathrm{X}_{\mathrm{S}}=\left[\mathrm{X}_{\mathrm{S}}(0), . ., \mathrm{X}_{\mathrm{S}}(\mathrm{M}-1)\right]$ to each relay node and to the destination. The received signal from satellite at the destination, with a channel noise $\mathrm{n}_{\mathrm{SD}}$, is represented as follow [5].

$$
Y_{S D}=\sqrt{\frac{P_{S}}{2}} * X_{S}+n_{S D}
$$

Similarly, the signal received at each relay node from the satellite after adding the noise $\mathrm{n}_{\mathrm{SR}}$ can be expressed mathematically [5]. 


$$
Y_{S R}=\sqrt{\frac{P_{S}}{2}} * X_{S}+n_{S R}
$$

In Phase II, the relay scale the receive signal represented in equation (2) in order to transmit a normalized vector $X_{R}$. If the instantaneous channel gain $\left|\mathrm{h}_{\mathrm{SR}}\right|^{2}$ and the retransmission power $P_{R}$ is known at the relay, the signals received at any relay are retransmitted with the gain $\beta$, considering the presence of the noise $\mathrm{N}_{0}[5]$.

$$
\beta=\sqrt{\frac{P_{R}}{P_{S}\left|h_{S R}\right|^{2}+N_{0}}}
$$

The received signal at the destination coming from any relay, after adding the channel noise $\mathrm{n}_{\mathrm{RD}}$, is calculated then according to the expression [5].

$$
Y_{R D}=\beta * h_{R D}+n_{R D}
$$

Finally, the received message is recovered through the Maximum Ratio Combiner MRC [6].

$$
Y_{M R C}=\sum_{i=1}^{k} h_{i d}^{*} * Y_{i d}
$$

The term $h_{i d}$ are the channel coefficients either from any relay to destination $\left(\mathrm{h}_{\mathrm{RD}}\right)$ or from satellite to the destination $\left(\mathrm{h}_{\mathrm{SD}}\right)$. Similarly, the term $\mathrm{Y}_{\mathrm{id}}$, is the signals from the relays to the destination $\left(\mathrm{Y}_{\mathrm{RD}}\right)$ or from the source to the destination $\left(\mathrm{Y}_{\mathrm{SD}}\right)$.

\section{B. Channel Model}

During the phase I, the satellite channel follow the free space model [7] [8]. The free space system model is based on the line of sight (LOS) component of the fading channel [7] and [8].

$$
H_{i j}=\alpha_{i j} * e^{\left(-j k_{0} f_{c} r_{i}\right)}
$$

The term $f_{c}$ is the carrier frequency, $r_{i}$ is the distance between the satellite and the $\mathrm{i}_{\text {th }}$ earth station (relay), $\mathrm{k}_{0}=$ $\left(2 * \pi / \mathrm{c}_{\mathrm{o}}\right)$ is the wave number and $\mathrm{c}_{\mathrm{o}}$ is the light speed. The phase $\varphi$ is assumed equal to zero and $\alpha$ (attenuation) is approximated to a constant [7].

$$
\alpha_{i j}=\frac{1}{2 k_{0} f_{c} r_{i}} * e^{(-j \varphi)}
$$

The phase II, is the phase for relaying the signals. Here each earth station transmits the amplified signal to the destination. The channel on earth between ground stations (relays) is assumed to follow a Rician distribution [9].

$$
\begin{gathered}
P(\gamma)=\left(\frac{\gamma}{\sigma^{2}}\right) e^{-\left(\frac{\gamma^{2}+s^{2}}{2 \sigma^{2}}\right) I_{0}\left(\frac{\gamma s}{\sigma^{2}}\right)} \\
s=\sqrt{m_{1}^{2}+m_{2}^{2}} \\
K=\frac{s^{2}}{2 \sigma^{2}}
\end{gathered}
$$

where $m_{1}$ and $m_{2}$ are two random variables mean. The term " $\mathrm{K}$ " is called Rician factor and $\sigma$ is the variance. Finally, $\mathrm{I}_{0}$ is the $0_{\text {th }}$ order Bessel Function.

\section{The Space Link}

For a transmitter with output of $\mathrm{P}_{\mathrm{t}}$ (watts) using a lossless antenna with gain $G_{t}$, the flux density in the direction of maximum power at a distance of $\mathrm{R}$ meters [10].

$$
\psi=\frac{P_{t} G_{t}}{4 \pi R^{2}}
$$

The product PtGt is often known as the effective isotropically radiated power (EIRP). The received power in the ground station [10].

$$
\begin{gathered}
P_{r}=\psi \mathrm{A}_{e f f} \\
A_{e f f}=\frac{\lambda^{2} G_{r}}{4 \pi} \\
P_{r}=\frac{E I R P}{4 \pi R^{2}} \frac{\lambda^{2} G_{r}}{4 \pi}=(E I R P)\left(G_{r}\right)\left(\frac{\lambda}{4 \pi R}\right)^{2}
\end{gathered}
$$

The term $(\lambda / 4 \pi R)^{2}$ is the path loss (FSL). It is the way how the energy is spread as an electromagnetic wave traveling from a source in three-dimensional space [11]. The equation in $\mathrm{dB}$ is represented [10].

$$
P_{r}=E I R P+G_{r}-F S L
$$

Knowing the distance to the satellite " $\mathrm{R}$ " in $\mathrm{Km}$ and the frequency "f" in $\mathrm{MHz}$, the free space losses are [10].

$$
F S L=32.4+20 \log (R)+20 \log (f)
$$

In satellite systems, the major source of noise on the equipment is called thermal noise and it comes from the random thermal motion of electrons in resistive and active devices in the reception path. The thermal noise power at the receiver [10].

$$
P_{n}=k T_{n} B_{n}
$$

The term $T_{n}$ is known as the equivalent noise temperature, $B_{n}$ is called the equivalent bandwidth of noise, and $k$ is Boltzmann's constant, where $k=1.38 \times 10-23 \mathrm{~J} / \mathrm{K}(228.6 \mathrm{~dB})$. The carrier to noise ratio is defined as the relation of the signal power received $\left(P_{r}\right)$ and the noise power $\left(P_{n}\right)$ [10].

$$
\frac{C}{N}=P_{r}-P_{n}
$$

Replacing (15) and (17) into (18)

$$
\begin{gathered}
\frac{C}{N}=E I R P+\frac{G}{T}-L-228.6-B \\
\frac{C}{N_{0}}=\frac{C}{N}+B
\end{gathered}
$$

\section{Simulation Results}

The simulation consists of a multiple relay integrated satellite-terrestrial cooperative system. The aim is transmitting signals in $\mathrm{Ka}$ band for the Venezuelan satellite 
into the Amazonian region. Basically, the main purpose of the simulation is to measure the BER performance over several transmissions. A randomly generated data stream of 1024 bits is transmitted over $10^{5}$ channel realizations.

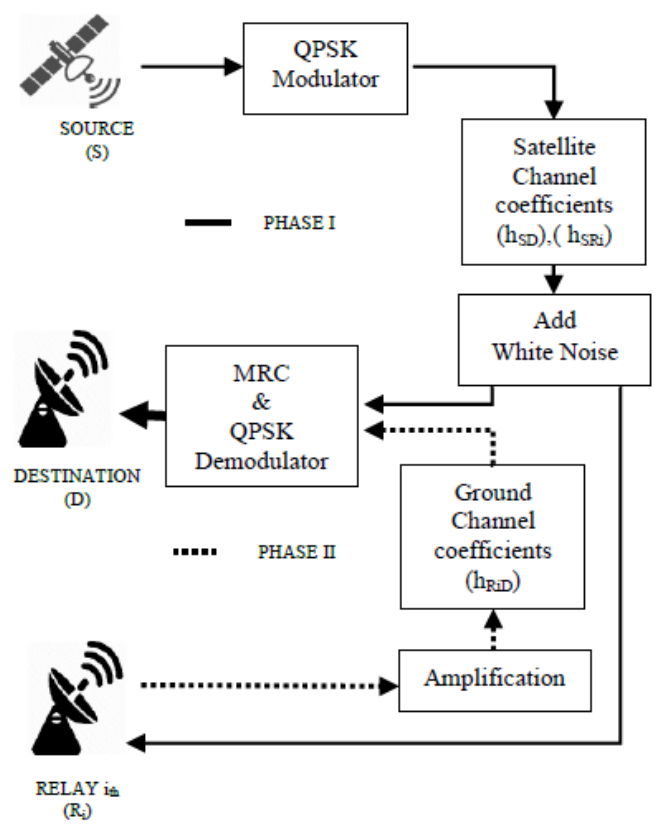

Fig. 2. VENESAT-1 AF cooperative diagram simulation.

During the Phase I, the satellite (source) transmit to earth a message of 1024 bits into a QPSK modulated signal. The transmitted signal over each channel is multiplied by the channel coefficients using (6) and immediately added the white noise. The channel coefficients and the Gaussian noise are randomly generated. For Ka band signals, the Venezuelan satellite use a carrier frequency around $19 \mathrm{GHz}$. The distance from the satellite to the ground station is about $36,000 \mathrm{Km}$. For the satellite link channel coefficients calculation, the simulation assumes the distance from the satellite to each earth stations varies about $\pm 3 \mathrm{Km}$. The signal received in the destination from the source is calculated using (1) and the signal received on $i_{\text {th }}$ relay station is represented by (2). The power of the source $P_{S}$ is the EIRP of the satellite. The free space losses are calculated by (16). The figure of merit G/T is set to $38.02 \mathrm{~dB}$ which is a real technical data into the Venezuelan ground station [12].

$$
P_{S}(d B)=E I R P=S N R-\frac{G}{T}+F S L+228.6
$$

The signal received coming from the satellite is amplified and forwarded in the second phase. The amplification factor is calculated using (3). The relay station transmission power is set to $P_{R}=10 \mathrm{~W}$. The channel coefficients and white noise are randomly generated. For terrestrial communication, the channel is considered as a Rician channel (8). In addition to the antenna gain, also exist other gains or attenuations in the reception path. The gain is provided by amplifiers and the attenuation are the losses in the waveguide, couplers or switches. For the simulation, a real reception gain of $158 \mathrm{~dB}$ is set according to the ground station specifications [12].

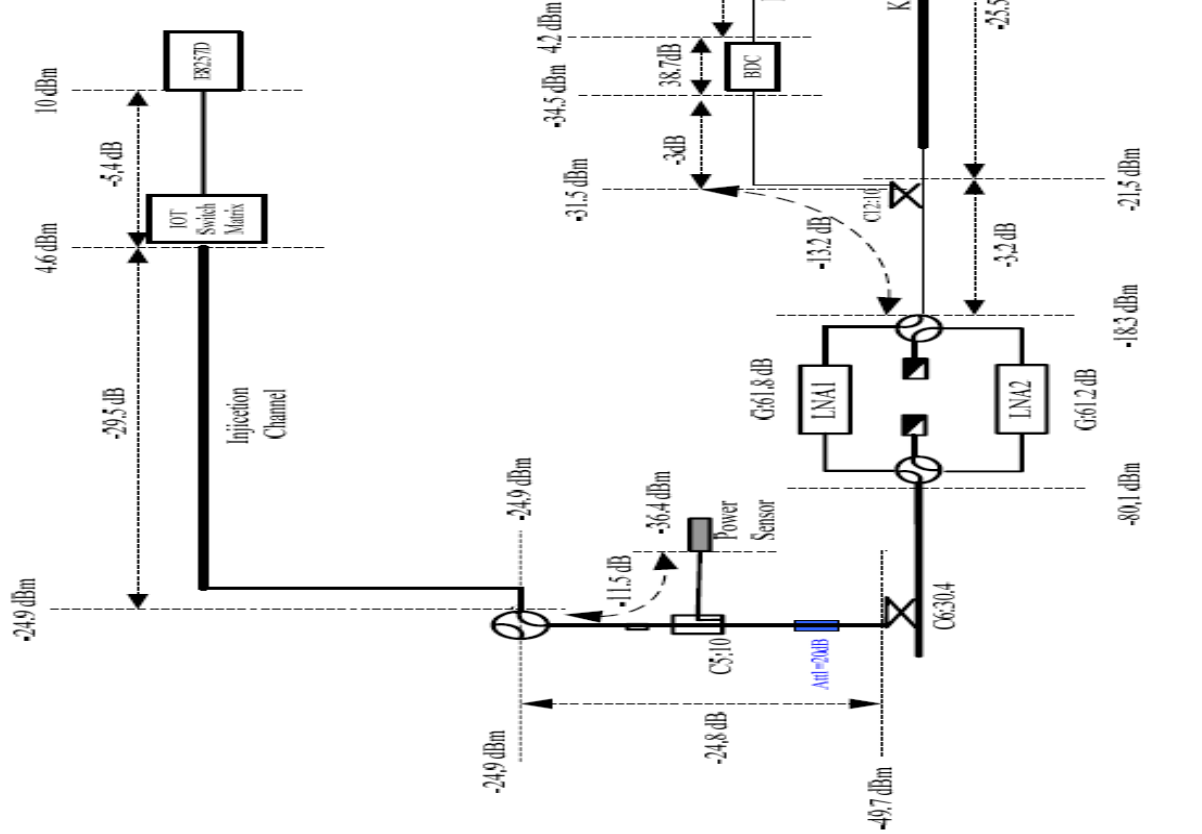

Fig. 3. VENESAT-1 Ka band ground station Rx gain path. 
The signal received in the destination is combined by the MRC using (5). The MRC output signal is demodulated and compared to the original 1024-bit data stream transmitted from the satellite. The number of errors are counted and the BER is calculated over the total of bit transmitted in $10^{5}$ channel realizations. Finally, the SNR needed to achieve $\mathrm{BER}=10^{-6}$ is compared for every case (multiple relaying or direct satellite communication).

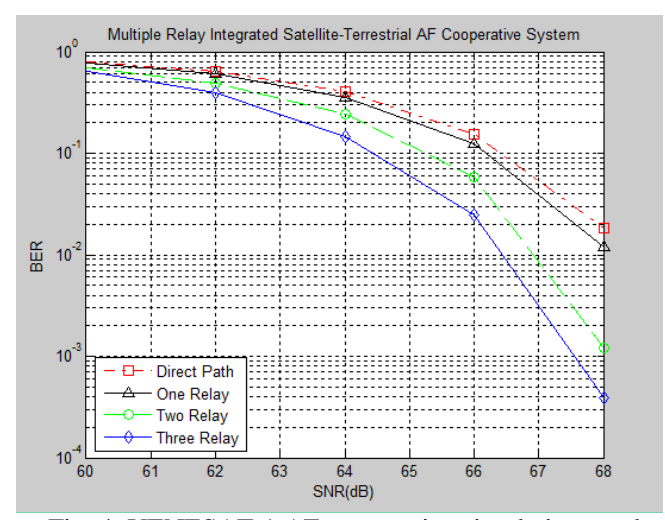

Fig. 4. VENESAT-1 AF cooperative simulation result.

The simulation result show that the Venezuelan satellite communication system in Ka Band, can be improved using a multiple relay integrated satellite-terrestrial cooperative system. For one relay, the gain improvement referred to the satellite direct path communication is not relevant. However, increasing the number of relay to more than one, the gain is significant. According to the simulation, with three relays, the reliability of the services increase about $2 \mathrm{~dB}$ of gain.

\section{CONCLUSION}

The signals in Ka band frequencies are sensitive to several climate phenomena, especially at the Amazon. The service providers need to consider the use of appropriate techniques, to keep the satellite link into acceptable levels of quality and reliability. A multiple relay integrated satellite-terrestrial cooperative system can improve the communication system performance of the Venezuelan satellite, increasing the reliability of the signal for various kinds of imperfect channel response such as those caused by noise or attenuation. For large attenuation channels, coding gain can be combined to the satellite-terrestrial cooperative system as a mitigation technique. Further studies should be done about the proposal impact on the information rate and bandwidth, in order to determine the most suitable applications than could be executed on the Amazonian region.

\section{REFERENCES}

[1] R. T. Schwarz, A. Knopp, D. Ogerman, C. A. Hofmann, and B. Lankl, "Optimun capacity MIMO satellite link for fixed and mobile services," in Proc. IEEE Workshop on Smart Antennas, 2008, vol. 1 pp. 209-216.

[2] ITU R P.618-11, "Propagation data and prediction methods required for the design of Earth-space telecommunication systems," (2013).

[3] T. Brown, E. D. Carvalho, and P. Kyritsi, Practical Guide to the MIMO Radio Channel, New York: Wiley 2012, ch. 2, pp. 15-26.

[4] M. Simon and M. Alouini, Digital Communication over Fading Channels, New York: Wiley, 2012.

[5] P. Hong, J. Huang, and J. Kuo, Cooperative Communications and Networking, New York: Springer, 2010, ch. 3, pp. 88-97.

[6] R. Liu, K. Sadek, W. Su, and A. Kawansiski, Cooperative Communications and Networking, New York: Canbridge University Press 2010, ch. 1, pp. 29-31.
[7] R. Adeogun, "Capacity and error rate analysis of MIMO satellite communication systems in fading scenarios," International Journal of Electrical and Computer Engineering (IJECE)

[8] C. Loo, "A statistical model for a land mobile satellite link," IEEE Transaction of Vehicular Technology, 1998 vol. 34, pp. 122-127.

[9] A. Giordano and H. Levesque, "Modelling of digital communication system using simulink," New Jersey: Wiley 2015, ch. 7, pp. 151-154.

[10] D. Rodis, Satellite Communications, New York: McGraw-Hill 2006, pp. 356-375.

[11] E. Lutz, M. Werner, and A. Jahn, Satellite Systems for Personal and Broadband Communications, Germany: Springer 2000, ch3 pp. 48-79.

[12] Station Hand Book for VENESAT-1 Satellite, 1st ed., Beijing Institute of Tracking and Telecommunication Technology, Beijing, 2008, pp. 23-40.

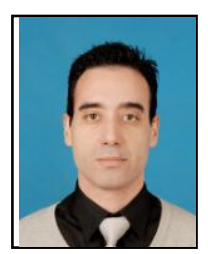

G. Guedez was born in Caracas Venezuela in 1978. In 2001 obtain the electronic engineer degree from the Venezuelan National Armed Forces University located at Maracay City, Aragua State, Venezuela. On March 2008 studied at Shenzou Institute of the China Academy of the Space Technology (CAST) located at Beijing, China, obtaining the space segment certificate. He has received the maintenance management master degree diploma conferred by the Venezuelan National Armed Forces University in 2011 and the master degree in electronic and communications systems in 2015 conferred by Beijing University of Aeronautics and Astronautics located at Beijing, China. His current and previous research interests are related to the mitigation techniques to improve the Venezuelan communication satellite system.

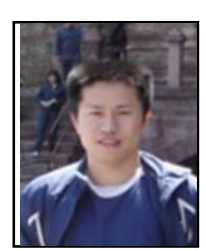

Q. Gao was born in China in 1971. September 1997 - July 2000: Ph.D., Institute of computing technology, Chinese Academy of Sciences, Beijing, China. September 1994 July 1997: MSc, Department of Physics, Lanzhou University, Lanzhou, China. September 1990 - July 1994: B.Sc., Department of Physics, Southwest China Normal University, Chongqing, China.

$\mathrm{He}$ is an associate professor/ professor at School of Electronic and Information Engineering in Beihang University, China, from 2004 until today. From October 2001 to October 2004 was working as a Research Fellow, at Department of Electronics Engineering, Aston University, UK. From February 2001- October 2001: Post doctor, National Research Center of Information Technology, Germany. His most recent publications are: "Impact of outdated channel state information on energy efficiency of co-operative hybrid automatic repeat request in wireless sensor networks", Wireless Sensor Systems, 2014 : 170-175, "Energy Hole Mitigation through Cooperative Transmission in Wireless Sensor Networks", International Journal of Distributed Sensor Networks, 2015:1-14 and "Outage-Optimal Relay Strategy under Outdated CSI in Decode-and-Forward Cooperative Communication Systems", IET Communications, 2015. His recent patents are: A MIMO Communication System Signal Detection Method, US 8831128B2, United States Patent and Channel Estimation Method, US 8934566B1, United States Patent. His current research interest is Wireless Communication and Wireless Networks and Data Mining.

Dr. Gao is a professor of School of Electronic and Information Engineering, Beihang University, Senior Expert of Regional Centre for Space Science and Technology Education in Asia and the Pacific in China (Affiliated to the United Nations), Senior Member of China Computer Federation and Senior Member of IEEE. His more relevant awards are: First-class Prize of National Defense Science and Technology Advancement in 2012, Winner of Education Ministry's New Century Excellent Talents supporting Plan in 2010 and Ad Hoc Networks Journal, Top Cited Article 2005-2010.

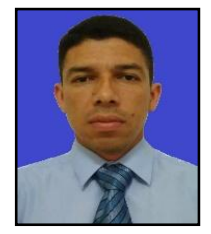

J. Uscategui was born in Táchira Venezuela in 1977. In 2000 obtained the systems engineer degree from Santiago Mariño Polytechnic University Institute located at San Cristóbal City, Táchira State, Venezuela. On March 2008 studied at Shenzou Institute of China Academy of the Space Technology (CAST) located at Beijing, China, obtaining the ground segment certificate about Radio Frequency subsystem. He has Science and Technology Management Specialist degree diploma conferred by the Dr. Rafael Belloso Chacin University in 2015 and the Master Degree in Project Management in 2015 conferred by Caribbean International University at Curaçao. His current and previous research interests are related to the Microsatellite Attitude and Determination Control System 